\title{
Adaptive Fuzzy Actuator Failure Compensation Control for a Class of MIMO Nonlinear Systems
}

\author{
Sabri BOULOUMA ${ }^{(1)^{*}}$, Salim LABIOD $^{(2)}$, Hamid BOUBERTAKH ${ }^{(3)}$ \\ (1) Unité de développement des Equipements Solaires, UDES/Centre de Développement des Energies \\ Renouvelables, CDER, Bou-Ismail, 42415, W. Tipaza, Algeria. \\ (2) LAJ, Faculty of Science and Technology, University of Jijel, Ouled Aissa, BP.98,1800, Jijel, Algeria \\ sab_blm@yahoo.fr
}

\begin{abstract}
In this paper, and adaptive fuzzy control scheme is developed for a class of uncertain multi-input multi-output (MIMO) nonlinear systems with uncertain actuator failures. Within this scheme, a fixed grouping of redundant actuators and a proportional actuation scheme for each group are considered. Afterward, a fuzzy system is used for the online approximation of a nominal control vector. The parameter adaptation laws for the nominal controller are driven by the prediction error, which is defined as the difference between the unknown nominal controller and the fuzzy controller. The proposed controller can compensate for both total and partial loss of effectiveness failure types. Closed-loop system stability and convergence properties of the tracking errors are proved based on Lyapunov theory. A piecewise analysis is carried out to prove that these properties hold despite the presence of parameter jumps caused by abrupt failures. A simulation study is carried out on a redundant joint two-link robot manipulator with joint failures at each link. Simulation results show the effectiveness of the proposed control scheme.
\end{abstract}

Keywords: Actuator failures, Fuzzy Systems, Adaptive Control, Lyapunov Stability, Redundant manipulator.

\section{INTRODUCTION}

Recently, actuator failure compensation has become an active area of research that has undergone remarkable progress. In the literature, various actuator failure compensation control techniques have been proposed. Generally, these techniques fall into two categories [1], [2]; passive approaches, where the controller is designed to be robust against a set of predefined failures. These approaches are generally based on the robust control theory which optimizes system's performance under actuator failures using $L Q R$ designs [3], $H_{\infty}$ designs [4] or using convex optimization based on linear matrix inequalities (LMI) [5]. These approaches are limited in that they deal only with a set of predefined failures [1]. On the other hand active failure compensation approaches are designed to react at the time of failure occurrence. Among these techniques, there are multiple model based designs [6], fault diagnosis and identification (FDI) based designs [2], neural networks and fuzzy logic based designs [4], [7] and adaptive control based designs [8]-[17].

Adaptive control updates the controller online to cope with different changes within the system, it has received considerable interest and successful applications in the area of actuator failure compensation [18], [19]. Numerous adaptive designs have been proposed for actuator failure compensation. A tutorial that summarizes the recent achievements in this field is given in [19]. The authors in [20] proposed adaptive control schemes of linear systems with lock in place actuator failures. In [16], [21], the authors propose an adaptive control scheme for nonlinear systems with unknown actuator failures using feedback linearization techniques. An adaptive backstepping control scheme for parametric strict feedback systems with actuator failures was proposed in [22]. In these works, system parameters are assumed known or partially known. The case of unknown nonlinear systems with unknown actuator failures has been rarely dealt with. In [8], [10], an adaptive fuzzy control scheme for uncertain nonlinear systems with actuator failures has been proposed for systems in the normal form. Backstepping and dynamic surface control (DSC) designs are proposed for parametric strict systems with actuator failures in [23]. In the aforementioned works, the idea was to bring the unknown system with unknown actuator failures into an ordinary system with unknown parameters, and then adaptive neural and fuzzy control techniques are applied to this system.

For MIMO nonlinear systems, a few works exist in this regard. In [19], [27], the authors proposed adaptive failure compensation control designs for MIMO systems with applications to aircraft and spacecraft. In[25], [32], adaptive actuator failure compensation for redundant robots and cooperating manipulators has been 
addressed,however these designs require knowledge ofthe system which is not always possible.

In this work, an adaptive fuzzy failure compensation controller is proposed for a class of uncertain nonlinear MIMO systems. This work extends the actuator failure compensation problem to the multivariable case which is more challenging compared to the single variable case, it also extends the actuator failure set where both partial and total loss of effectiveness are dealt with. Moreover, both the system and the failures are assumed unknown. Finally, theapproach consists only of a single controller which is approximated by a FLS to account for both system uncertainties and actuator failures. This is computationally effective compared tomultiple degrees of freedom controllers orFDI schemes. The rest of the paper is organized as follows: Section 2 outlines the problem and provides some preliminary analysis. Section 3 outlines the controller design and stability proofs. Section 4 presents a case study on a redundant joint two-link robot manipulator. Section 5 provides conclusions and perspectives.

\section{PROBLEM STATEMENT}

Consider the following class of MIMOsystems

$$
\begin{gathered}
y_{1}^{\left(r_{1}\right)}=f_{1}(\mathbf{x})+\sum_{j=1}^{q} g_{1 j}(\mathbf{x}) u_{j} \\
\vdots \\
y_{p}^{\left(r_{p}\right)}=f_{p}(\mathbf{x})+\sum_{j=1}^{q} g_{p j}(\mathbf{x}) u_{j}
\end{gathered}
$$

where $\mathbf{x}=\left[y_{1}, \ldots, y_{1}^{\left(r_{1}-1\right)}, \ldots, y_{p}, \ldots, y_{p}^{\left(r_{p}-1\right)}\right]^{T} \in \square^{n}$ is the state vector which is assumed available for measurements,the order of the system is $n=r_{1}+\ldots+r_{p}, \mathbf{u}=\left[u_{1}, \ldots, u_{q}\right]^{T} \in \square^{q}$ is the control input, $f_{i}(\mathbf{x})$ and $g_{i j}(\mathbf{x}), i=1, \ldots, p, j=1, \ldots, q$, $q>p$ are sufficiently smooth uncertain functions.

$$
\mathbf{y}^{(r)}=\left[y_{1}^{\left(r_{1}\right)}, \ldots, y_{p}^{\left(r_{p}\right)}\right]^{T}
$$

Then, system (1) can be written as follows

$$
\begin{aligned}
& \mathbf{y}^{(r)}=\mathbf{F}(\mathbf{x})+\mathbf{G}(\mathbf{x}) \mathbf{u} \\
& \text { with } \mathbf{F}(\mathbf{x})=\left(\begin{array}{l}
f_{1}(\mathbf{x}) \\
\vdots \\
f_{p}(\mathbf{x})
\end{array}\right), \mathbf{G}(\mathbf{x})=\left(\begin{array}{ccc}
g_{11}(\mathbf{x}) & \ldots & g_{1 q}(\mathbf{x}) \\
\vdots & \ddots & \vdots \\
g_{p 1}(\mathbf{x}) & \ldots & g_{p q}(\mathbf{x})
\end{array}\right)
\end{aligned}
$$

For the actuator failures, we consider two cases. In the first case, at time $t_{j}$, some inputs are no longer influenced by the control signal such that:

$$
u_{j}(t)=\bar{u}_{j}(t), t \geq t_{j}
$$

where $t_{j}$ is the failure time and $\bar{u}_{j}(t)$ the failure value.We assume that $\bar{u}_{j}$ can be expressed as

$$
\bar{u}_{j}(t)=\bar{u}_{j 0}+\sum_{l=1}^{n_{j}} \bar{u}_{j l} \delta_{j l}(t)
$$

for unknown constants $\bar{u}_{j l}$ and unknown smooth signals $\delta_{j l}(t), n_{j}$ is the number of $\delta_{j l}(t)$ in the $j^{\text {th }}$ failure pattern. A special case is when $\bar{u}_{j}(t)=\bar{u}_{j 0}$, this characterizes the lock in place failure type, i.e. at time $t_{j}$, the $j^{\text {th }}$ actuator is stuck at position $\bar{u}_{j 0}$. This type is common in practice, in an aircraft, for example, the flight control segments may be stuck at unknown positions during flight [18].

The second case is partial loss of effectiveness which is described mathematically as [9], [23]:

$$
u_{j}(t)=\rho_{j}(t) v_{j}(t), 0<\rho_{j}(t) \leq 1, t \geq t_{j}
$$

where $v_{j}(t)$ is the control signal to be designed.

In the presence of the two types (4) and/or (6) actuator failures, $\mathbf{u}(t)$ becomes

$$
\mathbf{u}(t)=\boldsymbol{\rho}(t) \mathbf{v}(t)+\boldsymbol{\sigma}(\overline{\mathbf{u}}(t)-\boldsymbol{\rho}(t) \mathbf{v}(t))
$$

where $\mathbf{v}(t)=\left[v_{1}(t), \ldots, v_{q}(t)\right]^{T}$,

$\boldsymbol{\rho}(t)=\operatorname{diag}\left\{\rho_{1}(t), \ldots, \rho_{q}(t)\right\}, \boldsymbol{\sigma}=\operatorname{diag}\left\{\sigma_{1}, \ldots, \sigma_{q}\right\}$, with $\sigma_{j}=1$ if the $j^{\text {th }}$ actuator fails as (4) and $\sigma_{j}=0$ otherwise.

The objective is to design a controller for the system (1), under unknown type (4) and/or type (6) actuator failures, to ensure stability and output tracking of a reference $y_{d}(t)=\left[y_{d 1}, \ldots, y_{d 2}\right]^{T}$. The derivatives of $y_{d i}(t)$ up to $r_{i}-1$ for $i=1, \ldots, p$ are assumed available and bounded. In this regard, we assume the following , [21].

Assumption 1. It is assumed that for up to $q-p$ type (4) actuator failures and up to $q$ type (6) actuator failures the system remains controllable.

This means that the remaining effective actuators should have the sufficient effect and power to achieve the control objective under all possible failure scenarios. In other words, assumption 1 implies that: (i) There is some actuation redundancy, i.e. there are actuators with the same effect. It is worth emphasizing 
that there should be at least $p$ effective actuators to keep the system controllable, otherwise the system will be under-actuated, and new control schemes will be necessary, (ii) Actuators with the same effects are arranged into $p$ groups with $m_{i}$ actuators in each group. For each group, there should be at least one remaining effective actuator.

For the adaptive control designand regarding system's redundancy, the following assumption of the control gain $\mathbf{G}(\mathbf{x})$ is made.

Assumption 2. The control gain matrix $\mathbf{G}(\mathbf{x})$ can be decomposed as: $\mathbf{G}(\mathbf{x})=\mathbf{G}_{\mathbf{0}}(\mathbf{x}) \mathbf{R}$, where $\mathbf{R} \in \square^{p \times q}$ is a known matrix and $\mathbf{G}_{\mathbf{0}}(\mathbf{x}) \in \square^{p \times p}$ is an unknown symmetric positive definite matrix.

Now, the following condition is set for system (1) to remain controllable despite actuator failures

$$
\operatorname{rank}\left(\left(\mathbf{I}_{\mathbf{q}}-\boldsymbol{\sigma}\right) \mathbf{R}^{\mathbf{T}}\right)=p
$$

From condition (8), it can be inferred that the matrix $\boldsymbol{R} \boldsymbol{\rho}(t)\left(\mathbf{I}_{\mathbf{q}}-\boldsymbol{\sigma}\right) \mathbf{R}^{T}$ is positive definite. Indeed, since $\quad \boldsymbol{\rho}(t)=\sqrt{\boldsymbol{\rho}(t)} \sqrt{\boldsymbol{\rho}(t)}^{T} \quad$ and. $\left(\mathbf{I}_{q}-\boldsymbol{\sigma}\right)=\left(\mathbf{I}_{q}-\boldsymbol{\sigma}\right)\left(\mathbf{I}_{q}-\boldsymbol{\sigma}\right)^{T}$, then, $\forall \mathbf{z} \in \square^{p}$, we have: $\mathbf{z}^{T}\left(R \boldsymbol{\rho}(t)\left(\mathbf{I}_{q}-\boldsymbol{\sigma}\right) \mathbf{R}^{T}\right) \mathbf{z}$

$=\mathbf{z}^{T}\left(\mathbf{R} \sqrt{\mathbf{\rho}(t)} \sqrt{\mathbf{\rho}(t)}^{T}\left(\mathbf{I}_{q}-\boldsymbol{\sigma}\right)\left(\mathbf{I}_{q}-\boldsymbol{\sigma}\right)^{T} \mathbf{R}^{T}\right) \mathbf{z}$

$$
=\mathbf{z}^{T}\left(\left(\mathbf{R} \sqrt{\boldsymbol{\rho}(t)}\left(\mathbf{I}_{q}-\boldsymbol{\sigma}\right)\right)\left(\left(\mathbf{I}_{q}-\boldsymbol{\sigma}\right)^{T} \sqrt{\boldsymbol{\rho}(t)}^{T} \mathbf{R}^{T}\right)\right) \mathbf{z}
$$

From (9), if $\left(\left(\mathbf{I}_{q}-\boldsymbol{\sigma}\right)^{T} \sqrt{\mathbf{\rho}(t)}^{T} \mathbf{R}^{T}\right) \mathbf{z}=\mathbf{0}$, the last term of (9) equals zero. From condition (8), $\left(\mathbf{I}_{q}-\boldsymbol{\sigma}\right)^{T} \mathbf{R}^{T}$ is full rank, i.e. its null space is limited to the trivial solution $z=0$. Thus $\mathbf{z}^{T}\left(\mathbf{R} \boldsymbol{\rho}(t)\left(\mathbf{I}_{q}-\boldsymbol{\sigma}\right) \mathbf{R}^{T}\right) \mathbf{z}>0$ for all $\mathbf{z} \neq 0$. Taking into account the redundant structure of the system and actuator's grouping, the following actuation scheme is proposed

$$
\mathbf{v}(t)=\mathbf{R}^{T} \mathbf{v}_{0}(t)
$$

In the presence of actuator failures, under the actuation scheme (10), equation (3) becomes

$$
\begin{aligned}
\mathbf{y}^{(r)} & =\mathbf{F}(\mathbf{x})+\mathbf{G}_{\mathbf{0}}(\mathbf{x}) \mathbf{R} \boldsymbol{\mathbf { u }} \overline{\mathbf{u}}(t) \\
& +\left(\mathbf{G}_{\mathbf{0}}(\mathbf{x}) \mathbf{R} \boldsymbol{\rho}(t)\left(\mathbf{I}_{q}-\boldsymbol{\sigma}\right) \mathbf{R}^{T}\right) \mathbf{v}_{0}(t)
\end{aligned}
$$

$$
\begin{gathered}
\overline{\mathbf{F}}(\mathbf{x})=\mathbf{F}(\mathbf{x})+\mathbf{G}_{\mathbf{0}}(\mathbf{x}) \mathbf{R} \boldsymbol{\sigma} \overline{\mathbf{u}}(t) \\
\overline{\mathbf{G}}(\mathbf{x})=\mathbf{G}_{\mathbf{0}}(\mathbf{x}) \mathbf{R \rho}(t)\left(\mathbf{I}_{q}-\boldsymbol{\sigma}\right) \mathbf{R}^{T}
\end{gathered}
$$

Then (11)can be rewritten more compactly as

$$
\mathbf{y}^{(r)}=\overline{\mathbf{F}}(\mathbf{x})+\overline{\mathbf{G}}(\mathbf{x}) \mathbf{v}_{\mathbf{0}}(t)
$$

Assumption3. Since $\mathbf{G}_{\mathbf{0}}(\mathbf{x})$ and $\boldsymbol{R} \boldsymbol{\rho}(t)\left(\mathbf{I}_{q}-\boldsymbol{\sigma}\right) \mathbf{R}^{T}$ are positive definite matrices, the matrix $\overline{\mathbf{G}}(\mathbf{x})=\mathbf{G}_{\mathbf{0}}(\mathbf{x}) \mathbf{R} \boldsymbol{\rho}(t)\left(\mathbf{I}_{q}-\boldsymbol{\sigma}\right) \mathbf{R}^{T}$ is assumed to remain positive definite under actuator failures occurrence, i.e. it is assumed that $\mathbf{z}^{T} \overline{\mathbf{G}}(\mathbf{x}) \mathbf{z}>0$ for all nonzero vectors $\mathbf{z} \in \square^{p}$. Besides, for proofs, it is assumed that $\underline{\lambda}\|\mathbf{z}\|^{2} \leq \mathbf{z}^{T} \overline{\mathbf{G}}(\mathbf{x}) \mathbf{z} \leq \bar{\lambda}\|\mathbf{z}\|^{2}$ where $\underline{\lambda}$ and $\bar{\lambda}$ are positive constants.

From (14), the faulty rectangular MIMO system is brought into a square MIMO system. In what follows, a controller will be designed for the system (14) to achieve the control objectives.

\section{ADAPTIVE CONTROLER DESIGN}

\subsection{Preliminary Analysis}

First, define the output tracking errors as follows

$$
e_{i}=y_{d i}(t)-y_{i}(t), i=1, \ldots, p
$$

Define also the filtered tracking errors as follows

$s_{i}(t)=\left(\frac{d}{d t}+\lambda_{i}\right)^{\left(r_{i}-1\right)} e_{i}(t), \lambda_{i}>0, i=1, \ldots, p$

From (16), $s_{i}(t), i=1, \ldots, p$ is a linear differential equation whose solution implies that the errors $e_{i}(t), i=1, \ldots, p$ and their derivatives up to $r_{i}-1$ converge to zero [33]. Thus, the control objective is to design a controller to keep $s_{i}(t), i=1, \ldots, p$ close to zero. Moreover, if $\left|s_{i}(t)\right| \leq \Phi_{i}$ where $\Phi_{i}$ is a positive constant, it follows that: $\left|e_{i}^{(j)}(t)\right| \leq 2^{j} \lambda_{i}^{j-r_{i}+1} \Phi_{i}, j=0, \ldots, r_{i}-1$ , $i=1, \ldots, p$. These bounds can be reduced by increasing $\lambda_{i}$.

The derivatives of the filtered tracking errors are

$$
\dot{s}_{i}=v_{i}-\overline{f_{i}}(\mathbf{x})-\sum_{j=1}^{p} \bar{g}_{i j}(\mathbf{x}) v_{0 j}(t), i=1, \ldots, p
$$

where $v_{1}, \ldots, v_{p}$ are defined as follows

$v_{i}=y_{d i}^{\left(r_{i}\right)}+\beta_{i, r i-1} e_{i}^{\left(r_{i}-1\right)}+\ldots+\beta_{i, 1} \dot{e}_{i}, i=1, \ldots, p$

Let us denote 
with $\beta_{i j}=C_{r_{i}-1}^{j-1} \lambda_{i}^{r_{i}-j}, C_{r_{i}-1}^{j-1}=\left(r_{i}-1\right) ! /\left(\left(r_{i}-j\right) !(j-1) !\right)$ for $\quad j=1, \ldots, r_{i}-1, i=1, \ldots, p . \quad \overline{f_{i}}(\mathbf{x}), \bar{g}_{i j}(\mathbf{x})$, $i, j=1, \ldots, p$ are the entries of $\overline{\mathbf{F}}(\mathbf{x})$ and $\overline{\mathbf{G}}(\mathbf{x})$ respectively. Let us denote $\mathbf{s}=\left[s_{1}, \ldots, s_{p}\right]^{T}$, $\mathbf{v}=\left[v_{1}, \ldots, v_{p}\right]^{T}$, then (17) can be written as

$$
\dot{\mathbf{s}}=\mathbf{v}-\overline{\mathbf{F}}(\mathbf{x})-\overline{\mathbf{G}}(\mathbf{x}) \mathbf{v}_{\mathbf{0}}(t)
$$

Now, choose the following ideal control law [34]

$$
\mathbf{v}_{\mathbf{0}}^{*}=\overline{\mathbf{G}}^{-1}(\mathbf{x})\left(-\overline{\mathbf{F}}(\mathbf{x})-\mathbf{v}+\mathbf{K s}+\mathbf{K}_{\mathbf{0}} \tanh \left(\frac{\mathbf{s}}{\varepsilon_{0}}\right)\right)
$$

with $\mathbf{K}=\operatorname{diag}\left\{k_{1}, \ldots, k_{p}\right\}, \mathbf{K}_{\mathbf{0}}=\operatorname{diag}\left\{k_{01}, \ldots, k_{0 p}\right\}$, and $k_{i}, k_{0 i}>0$, for $i=1, \ldots, p, \varepsilon_{0}$ is a small positive constant, $\tanh ($.$) stands for the$ hyperbolic tangent function defined for the vector s as $\tanh \left(\mathbf{s} / \varepsilon_{0}\right)=\left[\tanh \left(s_{1} / \varepsilon_{0}\right), \ldots, \tanh \left(s_{p} / \varepsilon_{0}\right)\right]^{T}$.

In fact, substituting (20) into (19) yields

$$
\dot{\mathbf{s}}=-\mathrm{Ks}-\mathrm{K}_{0} \tanh \left(\frac{\mathbf{s}}{\varepsilon_{0}}\right)
$$

From (21) it can be concluded that $\lim _{t \rightarrow \infty} s_{i}(t)=0$ and $\lim _{t \rightarrow \infty} e_{i}^{(j)}(t)=0, j=0, \ldots, r_{i}-1, i=1, \ldots, p$.

The control law (20) is not implementable since $\overline{\mathbf{F}}(\mathbf{x})$ and $\overline{\mathbf{G}}(\mathbf{x})$ are unknown. Therefore, a FLS is used to adaptively build the control law (20).

\section{A. Adaptive Fuzzy Controller Design}

Let us denote $\mathbf{v}_{\mathbf{0}}^{*}=\left[v_{01}^{*}, \ldots, v_{0 p}^{*}\right]^{T}$ the ideal control law (20). Assume that each entry of $\mathbf{v}_{0}^{*}$ can be approximated by a fuzzy system $v_{0 i}$ as

$$
v_{0 i}=\mathbf{w}_{i}^{T}(\mathbf{z}) \boldsymbol{\theta}_{i}
$$

where $\mathbf{w}_{i}(\mathbf{z})$ is a fuzzy regressor and $\boldsymbol{\theta}_{\mathbf{i}}$ is a parameter. The optimal approximation of $v_{0 i}^{*}$ is

$$
v_{0 i}^{*}=\mathbf{w}_{i}^{T}(\mathbf{z}) \boldsymbol{\theta}_{i}^{*}+\varepsilon_{i}(\mathbf{z})
$$

let $\boldsymbol{\varepsilon}(\mathbf{z})=\left[\varepsilon_{1}(\mathbf{z}), \ldots, \varepsilon_{p}(\mathbf{z})\right]^{T}, \quad \boldsymbol{\theta}=\left[\boldsymbol{\theta}_{1}^{T}, \ldots, \boldsymbol{\theta}_{p}^{T}\right]$, and $\mathbf{w}(\mathbf{z})=\operatorname{diag}\left\{\mathbf{w}_{\mathbf{1}}(\mathbf{z}), \ldots, \mathbf{w}_{\mathbf{p}}(\mathbf{z})\right\}$. Then (22) and (23) can be written as follows

$$
\begin{gathered}
\mathbf{v}_{\mathbf{0}}=\mathbf{w}^{\mathrm{T}}(\mathbf{z}) \boldsymbol{\theta} \\
\mathbf{v}_{\mathbf{0}}^{*}=\mathbf{w}^{T}(\mathbf{z}) \boldsymbol{\theta}^{*}+\boldsymbol{\varepsilon}(\mathbf{z})
\end{gathered}
$$

Define the prediction error vector $\mathbf{e}_{\mathbf{v}_{0}}$ as follows

$$
\mathbf{e}_{\mathbf{v}_{0}}=\mathbf{v}_{0}^{*}-\mathbf{v}_{0}
$$

Using (24) and (25), (26) can be written as:

$$
\mathbf{e}_{\mathbf{v}_{0}}=\mathbf{w}^{T}(\mathbf{z}) \tilde{\boldsymbol{\theta}}+\boldsymbol{\varepsilon}(\mathbf{z})
$$

where $\tilde{\boldsymbol{\theta}}=\boldsymbol{\theta}^{*}-\boldsymbol{\theta}$. Using (19) and (27), the time derivative of $\mathbf{s}$ can written as follows

$$
\dot{\mathbf{s}}=-\mathbf{K s}-\mathbf{K}_{\mathbf{0}} \tanh \left(\frac{\mathbf{s}}{\varepsilon_{0}}\right)+\overline{\mathbf{G}}(\mathbf{x}) \mathbf{e}_{\mathrm{v}_{\mathbf{0}}}
$$

Based on the gradient descentmethod with a $\sigma-$ modification[34], we propose the following robust parameter adaptation law

$$
\dot{\boldsymbol{\theta}}=\eta \mathbf{w}(\mathbf{z}) \overline{\mathbf{G}}(\mathbf{x}) \mathbf{e}_{\mathbf{v}_{0}}-\eta \sigma \boldsymbol{\theta}
$$

where $\eta$ is the adaptation gain and $\sigma$ is a small positive constant. In (29), the term $e_{v_{0}}$ is not available, however, from (28) the term $\overline{\mathbf{G}}(\mathbf{x}) \mathbf{e}_{\mathbf{v}_{0}}$ can be pulled and substituted into (33), yielding

$$
\dot{\boldsymbol{\theta}}=\eta \mathbf{w}(\mathbf{z})\left(\dot{\mathbf{s}}+\mathbf{K s}+\mathbf{K}_{\mathbf{0}} \tanh \left(\frac{\mathbf{s}}{\varepsilon_{0}}\right)\right)-\eta \sigma \boldsymbol{\theta}
$$

In the following closed-loop system stability and tracking proofs are presented.

\section{B. Stability and Tracking Analysis}

Suppose that failures occur at time instants $t_{k}, k=0,1, \ldots, N$ where $0=t_{0}<\ldots<t_{N}<\infty$. At each time $t_{k}$, one or more actuators fail or recover from a failure. Consider a stepwise positive function defined over the time interval $t \in\left[t_{k}, t_{k+1}\right), k=0, \ldots, N$ as follows

$$
V_{\boldsymbol{\theta}, k}=\frac{1}{2 \eta} \tilde{\boldsymbol{\theta}}^{T} \tilde{\boldsymbol{\theta}}
$$

Differentiating (31) over the interval $t \in\left[t_{k}, t_{k+1}\right)$ and using (28), (27) and(30) yields

$$
\dot{V}_{\boldsymbol{\theta}, k}=-\mathbf{e}_{\mathbf{v}_{\mathbf{0}}}^{T} \overline{\mathbf{G}}(\mathbf{x}) \mathbf{e}_{\mathbf{v}_{\mathbf{0}}}+\boldsymbol{\varepsilon}^{T}(\mathbf{z}) \overline{\mathbf{G}}(\mathbf{x}) \mathbf{e}_{\mathbf{v}_{\mathbf{0}}}-\sigma \tilde{\boldsymbol{\theta}}^{T} \boldsymbol{\theta}
$$

Now, by virtue of the following inequalities

$$
\begin{gathered}
\sigma \tilde{\boldsymbol{\theta}}^{T} \boldsymbol{\theta} \leq-\frac{\sigma}{2}\|\tilde{\boldsymbol{\theta}}\|^{2}+\frac{\sigma}{2}\left\|\boldsymbol{\theta}^{*}\right\|^{2} \\
\boldsymbol{\varepsilon}^{T}(\mathbf{z}) \overline{\mathbf{G}}(\mathbf{x}) \mathbf{e}_{\mathbf{v}_{\mathbf{0}}} \leq \mathbf{e}_{\mathbf{v}_{\mathbf{0}}}^{T}(\mathbf{z}) \overline{\mathbf{G}}(\mathbf{x}) \mathbf{e}_{\mathbf{v}_{\mathbf{0}}}+\boldsymbol{\varepsilon}^{T}(\mathbf{z}) \overline{\mathbf{G}}(\mathbf{x}) \boldsymbol{\varepsilon}(\mathbf{z})
\end{gathered}
$$

Equation (32) can be bounded as follows

$$
\begin{aligned}
& \dot{V}_{\boldsymbol{\theta}, k} \leq-\frac{3}{4} \mathbf{e}_{\mathbf{v}_{\mathbf{0}}}^{T}(\mathbf{z}) \overline{\mathbf{G}}(\mathbf{x}) \mathbf{e}_{\mathbf{v}_{\mathbf{0}}}-\boldsymbol{\varepsilon}^{T}(\mathbf{z}) \overline{\mathbf{G}}(\mathbf{x}) \mathbf{e}_{\mathbf{v}_{\mathbf{0}}} \\
& -\frac{\sigma}{2}\|\tilde{\boldsymbol{\theta}}\|^{2}+\boldsymbol{\varepsilon}^{T}(\mathbf{z}) \overline{\mathbf{G}}(\mathbf{x}) \boldsymbol{\varepsilon}(\mathbf{z})+\frac{\sigma}{2}\left\|\boldsymbol{\theta}^{*}\right\|^{2}
\end{aligned}
$$


By virtue of assumption 3 , we can write:

$$
\boldsymbol{\varepsilon}^{T} \overline{\mathbf{G}}(\mathbf{x}) \boldsymbol{\varepsilon} \leq \bar{\lambda} \boldsymbol{\varepsilon}^{T} \boldsymbol{\varepsilon}
$$

Since $\boldsymbol{\theta}^{*}$ is constant over a fixed failure interval $\left[t_{k}, t_{k+1}\right)$ and $\boldsymbol{\varepsilon}(\mathbf{z})$ is assumed bounded. A positive bound $\psi_{k}$ can be defined over $\left[t_{k}, t_{k+1}\right)$ as follows

$$
\psi_{k}=\sup _{\boldsymbol{z} \in \Omega}\left(\bar{\lambda} \boldsymbol{\varepsilon}^{T} \boldsymbol{\varepsilon}+\frac{\sigma}{2}\left\|\boldsymbol{\theta}^{*}\right\|^{2}\right)
$$

Hence, (35) can be further simplified as follows

$\dot{V}_{\boldsymbol{\theta}, k} \leq-\alpha V_{\boldsymbol{\theta}, k}-\frac{3}{4} \mathbf{e}_{\mathbf{v}_{\mathbf{0}}}^{T} \overline{\mathbf{G}}(\mathbf{x}) \mathbf{e}_{\mathbf{v}_{\mathbf{0}}}+\psi_{k} \leq-\alpha V_{\boldsymbol{\theta}, k}+\psi_{k}$

where $\alpha=\sigma \eta$. We can prove the following theorem on the boundedness of the error vector $\tilde{\boldsymbol{\theta}}$.

Theorem 1. Under assumptions 1,2 and 3 , if the condition (8) is met, the control signal (24) with adaptation law (30) and the actuation scheme (10) applied to the system (1) subject to actuator failures guarantees the following closed-loop properties:

- The parameter error vector $\tilde{\boldsymbol{\theta}}$ is bounded and converges to a residual set $\Omega_{\boldsymbol{\theta}}=\left\{\tilde{\boldsymbol{\theta}}:\|\tilde{\boldsymbol{\theta}}\|^{2} \leq 2 \eta \psi / \alpha+\sum_{k=1}^{N}\left\|\Delta \tilde{\boldsymbol{\theta}}\left(t_{k}\right)\right\|^{2}\right\} \quad$ after $t_{N}$ where no further actuator failures occur.

- The control error vector $\mathbf{e}_{\mathbf{v}_{0}}$ is bounded and it is $\left(\bar{\varepsilon}^{2}+\sigma\right)$ small in the mean square sense.

Proof : Equation (38) means that for $V_{\boldsymbol{\theta}, k} \geq \psi_{k} / \alpha, \quad \dot{V}_{\boldsymbol{\theta}, k}<0$.Hence, $\quad V_{\boldsymbol{\theta}, k}$ and $\tilde{\boldsymbol{\theta}}$ are bounded over the interval $\left[t_{k}, t_{k+1}\right)$ given that jumps due to actuator failures are bounded. In addition, since $\varepsilon(\mathbf{z}), \mathbf{w}(\mathbf{z})$ are assumed bounded, it follows that $\mathbf{e}_{\mathbf{v}_{0}}$ is bounded over $\left[t_{k}, t_{k+1}\right)$. Integrating (38) over $\left[t_{k}, t_{k+1}\right)$, yields

$$
\|\tilde{\boldsymbol{\theta}}(t)\|^{2} \leq\left\|\tilde{\boldsymbol{\theta}}\left(t_{k}\right)\right\|^{2} e^{-\alpha\left(t-t_{k}\right)}+\frac{2 \eta \psi_{k}}{\alpha}
$$

Now, due to actuator failures, $V_{\theta}$ and, consequently $\tilde{\boldsymbol{\theta}}$ exhibit jumps at time instants $t_{k}, k=1, \ldots, N$. Let us denote it as $\Delta \tilde{\boldsymbol{\theta}}\left(t_{k}\right)=\tilde{\boldsymbol{\theta}}\left(t_{k}^{+}\right)-\tilde{\boldsymbol{\theta}}\left(t_{k}^{-}\right)$, where $t_{k}^{-}, t_{k}^{+}$are the instants just before and after the failure occurrence respectively. Thus, we can write

$\left\|\tilde{\boldsymbol{\theta}}\left(t_{k}^{+}\right)\right\|^{2}=\left\|\tilde{\boldsymbol{\theta}}\left(t_{k}^{-}\right)+\Delta \tilde{\boldsymbol{\theta}}\left(t_{k}\right)\right\|^{2} \leq\left\|\Delta \tilde{\boldsymbol{\theta}}\left(t_{k}\right)\right\|^{2}+\left\|\tilde{\boldsymbol{\theta}}\left(t_{k}^{-}\right)\right\|^{2}(40)$

Starting from $t_{1}$, we have

$$
\left\|\tilde{\boldsymbol{\theta}}\left(t_{1}^{+}\right)\right\|^{2} \leq\left\|\tilde{\boldsymbol{\theta}}\left(t_{1}^{-}\right)\right\|^{2}+\left\|\Delta \tilde{\boldsymbol{\theta}}\left(t_{1}\right)\right\|^{2}
$$

Now, from (39), we can write

$$
\left\|\tilde{\boldsymbol{\theta}}\left(t_{k}^{-}\right)\right\|^{2} \leq\left\|\tilde{\boldsymbol{\theta}}\left(t_{0}\right)\right\|^{2} e^{-\alpha t}+\frac{2 \eta \psi_{0}}{\alpha} \leq\left\|\tilde{\boldsymbol{\theta}}\left(t_{0}\right)\right\|^{2}+\frac{2 \eta \psi_{0}}{\alpha}
$$

Substituting (42) into (41) and taking $k=1$ yields

$$
\left\|\tilde{\boldsymbol{\theta}}\left(t_{1}^{+}\right)\right\|^{2} \leq\left\|\tilde{\boldsymbol{\theta}}\left(t_{0}\right)\right\|^{2}+\frac{2 \eta \psi_{1}}{\alpha}+\left\|\Delta \tilde{\boldsymbol{\theta}}\left(t_{1}\right)\right\|^{2}
$$

Likewise, we proceed for $t_{2}, \ldots, t_{N}$, at $t_{N}$, we obtain

$\left\|\tilde{\boldsymbol{\theta}}\left(t_{N}^{+}\right)\right\|^{2} \leq\left\|\tilde{\boldsymbol{\theta}}\left(t_{0}\right)\right\|^{2}+\frac{2 \eta}{\alpha} \sum_{k=1}^{N} \psi_{k}+\sum_{k=1}^{N}\left\|\Delta \tilde{\boldsymbol{\theta}}\left(t_{k}\right)\right\|^{2}$

Now, by integrating (38) over $\left[t_{N}, t\right)$ yields

$$
\begin{aligned}
& \|\tilde{\boldsymbol{\theta}}(t)\|^{2} \leq\left\|\tilde{\boldsymbol{\theta}}\left(t_{N}^{+}\right)\right\|^{2} e^{-\alpha\left(t-t_{N}\right)} \\
& +\frac{2 \eta}{\alpha} \sum_{k=1}^{N} \psi_{k}+\sum_{k=1}^{N}\left\|\Delta \tilde{\boldsymbol{\theta}}\left(t_{k}\right)\right\|^{2}
\end{aligned}
$$

Equation (45) implies that $\|\tilde{\boldsymbol{\theta}}(t)\|^{2}$ is bounded for $t \in\left[t_{k}, t_{k+1}\right)$. Besides, as $t \rightarrow \infty, \quad\|\tilde{\boldsymbol{\theta}}(t)\|^{2}$ converges to a residual set $\Omega_{\theta}$ defined as

$$
\Omega_{\boldsymbol{\theta}}=\left\{\tilde{\boldsymbol{\theta}}:\|\tilde{\boldsymbol{\theta}}\|^{2} \leq 2 \eta \psi / \alpha+\sum_{k=1}^{N}\left\|\Delta \tilde{\boldsymbol{\theta}}\left(t_{k}\right)\right\|^{2}\right\}
$$

Now, given that $\|\tilde{\boldsymbol{\theta}}(t)\|^{2}, V_{\boldsymbol{\theta}}$ and $\mathbf{e}_{\mathbf{v}_{0}} \in L_{\infty}$. From assumption 3 , one has

$$
\underline{\lambda} \mathbf{e}_{\mathbf{v}_{0}}^{T} \mathbf{e}_{\mathbf{v}_{0}} \leq \mathbf{e}_{\mathbf{v}_{0}}^{T} \overline{\mathbf{G}}(\mathbf{x}) \mathbf{e}_{\mathbf{v}_{0}} \leq \bar{\lambda} \mathbf{e}_{\mathbf{v}_{0}}^{T} \mathbf{e}_{\mathbf{v}_{0}}
$$

Let $\|\boldsymbol{\varepsilon}(\mathbf{z})\|<\bar{\varepsilon}$,integrate(47) for $t \in[t, t+T)$ yields

$$
\begin{aligned}
& \int_{t}^{t+T}\left\|\mathbf{e}_{\mathbf{v}_{0}}\right\|^{2} d \tau \leq \frac{4}{3 \underline{\lambda}}\left(\bar{\lambda} \bar{\varepsilon}^{2}+\frac{\sigma}{2}\left\|\boldsymbol{\theta}^{*}\right\|^{2}\right) T \\
& +\frac{4}{3 \underline{\lambda}}\left(V_{\boldsymbol{\theta}}(t)-V_{\boldsymbol{\theta}}(t+T)\right)
\end{aligned}
$$

Which can be bounded as

$$
\int_{t}^{t+T}\left\|\mathbf{e}_{\mathbf{v}_{0}}\right\|^{2} d \tau \leq c_{1}\left(\bar{\varepsilon}^{2}+\sigma\right) T+c_{2}
$$

with $c_{1}=\frac{4}{3 \underline{\lambda}} \max \left(\bar{\lambda},\left\|\boldsymbol{\theta}^{*}\right\| / 2\right), c_{2}=\frac{4}{3 \underline{\lambda}} \sup _{t}\left(V_{\boldsymbol{\theta}}(t)-V_{\boldsymbol{\theta}}(t+T)\right)$. Equation (49) implies $\left\|\mathbf{e}_{\mathbf{v}_{0}}\right\|$ is $\left(\bar{\varepsilon}^{2}+\sigma\right)$-small in the mean square sense.

\subsection{Tracking error convergence analysis}

Consider the following Lyapunov-like function

$$
V_{\mathrm{s}}=\frac{1}{2} \mathbf{s}^{T} \mathbf{s}
$$

Using (27) and (28), $\dot{V}_{\mathrm{s}}$ simplifies as follows 


$$
\dot{V}_{\mathbf{s}}=\mathbf{s}^{\mathrm{T}} \mathbf{K} \mathbf{s}-\mathbf{s}^{\mathrm{T}} \mathbf{K}_{\mathbf{0}} \tanh \left(\frac{\mathbf{s}}{\varepsilon_{0}}\right)+\mathbf{s}^{\mathrm{T}} \overline{\mathbf{G}}(\mathbf{x})\left(\mathbf{w}^{\mathrm{T}}(\mathbf{z}) \tilde{\boldsymbol{\theta}}+\boldsymbol{\varepsilon}(\mathbf{z})\right)
$$

From (45), we have

$$
\|\tilde{\boldsymbol{\theta}}(t)\| \leq\left\|\tilde{\boldsymbol{\theta}}\left(t_{N}^{+}\right)\right\| e^{-0.5 \alpha\left(t-t_{N}\right)}+\sqrt{\frac{2 \eta \psi_{N+1}}{\alpha}}
$$

Since $\mathbf{w}(\mathbf{z}), \boldsymbol{\varepsilon}(\mathbf{z}), \overline{\mathbf{G}}(\mathbf{x})$ are bounded, we have

$$
\left\|\overline{\mathbf{G}}(\mathbf{x})\left(\mathbf{w}^{T}(\mathbf{z}) \tilde{\boldsymbol{\theta}}\right)+\boldsymbol{\varepsilon}(\mathbf{z})\right\| \leq \phi_{1} e^{-0.5 \alpha t}+\phi_{2}
$$

where $\phi_{1}$ and $\phi_{2}$ are positive constants. By virtue of (53), (51) can be bounded as follows

$$
\dot{V_{\mathbf{s}}} \leq-\mathbf{s}^{\mathrm{T}} \mathbf{K s}-\mathbf{s}^{\mathrm{T}} \mathbf{K}_{\mathbf{0}} \tanh \left(\frac{\mathbf{s}}{\varepsilon_{0}}\right)+\|\mathbf{s}\|\left(\phi_{1} e^{-0.5 \alpha t}+\phi_{2}\right)
$$

Given that $\|\mathbf{s}\| \leq \sum_{i=1}^{p}\left|s_{i}\right|$, (54) can be written as

$$
\begin{aligned}
\dot{V_{\mathrm{s}} \leq} & -\sum_{i=1}^{p} k_{i} s_{i}^{2}-\sum_{i=1}^{p} k_{0 i} s_{i} \tanh \left(\frac{s_{i}}{\varepsilon_{0}}\right) \\
& +\sum_{i=1}^{p} \phi_{1}\left|s_{i}\right| e^{-0.5 \alpha t}+\sum_{i=1}^{p} \phi_{2}\left|s_{i}\right|
\end{aligned}
$$

ifthe free design parameters $k_{0 i}$ are chosen such that $k_{0 i}>\phi_{1}$, using the inequality [29]: $-s_{i} \tanh \left(s_{i} / \varepsilon_{0}\right)+\left|s_{i}\right| \leq \kappa \varepsilon_{0}, \kappa=0.2785$. Inequality (55) can be further simplified as follows:

$$
\dot{V}_{\mathrm{s}} \leq-\sum_{i=1}^{p} k_{i} s_{i}^{2}+\sum_{i=1}^{p} \phi_{1}\left|s_{i}\right| e^{-0.5 \alpha t}+p \phi_{2} \kappa \varepsilon_{0}
$$

With $\phi_{1}\left|s_{i}\right| e^{-0.5 \alpha t} \leq 0.25 s_{i}^{2}+\phi_{1}^{2} e^{-0.5 \alpha t},(56)$ gives

$$
\dot{V}_{\mathrm{s}} \leq-\sum_{i=1}^{p}\left(k_{i}-0.25\right) s_{i}^{2}+p \phi_{2} \kappa \varepsilon_{0}
$$

where $k_{i}$ are chosen such that $k_{i}>0.25$. Now, letting $\alpha_{\mathrm{s}}=2 \min _{1 \leq i \leq p}\left(k_{i}-0.25\right)$, (57) simplifies to

$$
\dot{V_{\mathrm{s}}} \leq-\alpha_{\mathrm{s}} V_{\mathrm{s}}+p \phi_{1}^{2} e^{-\alpha t}+p \phi_{2} \kappa \varepsilon_{0}
$$

Now we can prove the following theorem:

Theorem 2. The control law (24)with the adaptation law (30) and actuation scheme (10) guarantees the boundedness of the signals $x$ and $v_{0}$. In addition, the tracking errors are asymptotically bounded as: $\left|e_{i}^{(t)}(t)\right| \leq 2^{j} \lambda_{i}^{j-r_{i}+1} \Phi$, for with $\Phi=\sqrt{2 p \phi_{2} \kappa \varepsilon_{0} / \alpha_{\mathrm{s}}}$.

Proof. Equation (58) implies that, for .., $\dot{V}<0$. Therefore, $\mathbf{s}(t)$ is bounded. Given that $y_{d}(t)$ and its derivatives are bounded, it follows that $\mathbf{x}$ is bounded. Moreover, since the term $p \phi_{1}^{2} e^{-\alpha t}$ converges to zero as $t \rightarrow \infty, V$, converges to a bound defined as: $V_{\mathrm{s}}<2 p \phi_{2} \kappa \varepsilon_{0} / \alpha_{\mathrm{s}}$. Therefore, $\mathbf{s}(t)$ will converge asymptotically to a residual set ... This implies that the tracking errors and their derivatives are asymptotically bounded as $\left|e_{i}^{(j)}(t)\right| \leq 2^{j} \lambda_{i}^{j-r_{i}+1} \Phi$, for $j=1, \ldots, r_{i}-1, i=1, \ldots, p$. This bound can be reduced by tuning the design parameters $k_{i}, \lambda_{i}$ and $\varepsilon_{0}$.On the other hand, from theorem $1, \tilde{\boldsymbol{\theta}}, \boldsymbol{\theta} \in L_{\infty}$. Since $\mathbf{w}(\mathbf{z})$ is bounded, (24) implies that $\mathbf{v}_{0}$ is bounded.

\section{CASE STUDY}

In this section, simulation is carried out on a manipulator with redundant actuation with the proposed actuator failure compensation scheme. In this system, the torque is provided by concurrently actuated joints. This provides redundancy, in case of a joint failure; the control effort is redistributed among healthy joints. In practice, when a joint fails, it will be disengaged by a clutch to counteract to torque (ङ25plied by healthy actuators, see Figure1 [32].

The dynamic model of a concurrently actuated two-link robot manipulator is formulated as [32]

$$
\mathbf{D}(\mathbf{q}) \ddot{\mathbf{q}}+\mathbf{C}(\mathbf{q}, \dot{\mathbf{q}}) \dot{\mathbf{q}}+\mathbf{g}(\mathbf{q})=\boldsymbol{\tau}
$$

where $\mathbf{q}, \dot{\mathbf{q}}, \ddot{\mathbf{q}} \in \square^{2 \times 1}$ are the joint angular position, velocity, and acceleration respectively.

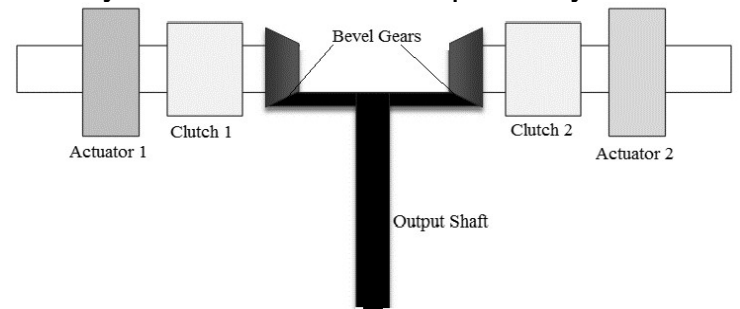

Figure 1. Example of a dual actuation system where $\mathbf{D}(\mathbf{q}) \in \square^{2 \times 2}$ is the manipulator's inertia matrix, $\quad \mathbf{C}(\mathbf{q}, \dot{\mathbf{q}}) \in \square^{2 \times 2}$ is the Coriolis and centrifugal term, $\mathbf{g}(\mathbf{q}) \in \square^{2 \times 1}$ is the gravity term, more details on the formulation of these terms can be found on [28], $\tau \in \square^{2 \times 1}$ is the torque vector.

For a manipulator with redundant joints, at the $i^{\text {th }}$ joint, $i=1,2$, there are $m_{i}$ actuators operating concurrently to provide the required torque. For the $i^{\text {th }}$ joint, the torque is formulated as

$$
\tau_{i}=\tau_{i 1}+\ldots+\tau_{i m_{i}}
$$

In the dual actuation case, the corresponding torque vector can be written as 


$$
\boldsymbol{\tau}=\left(\begin{array}{l}
\tau_{1} \\
\tau_{2}
\end{array}\right)=\left(\begin{array}{llll}
1 & 1 & 0 & 0 \\
0 & 0 & 1 & 1
\end{array}\right)\left(\begin{array}{l}
\tau_{11} \\
\tau_{12} \\
\tau_{21} \\
\tau_{22}
\end{array}\right)
$$

The dynamic model of an ordinary two-link manipulator (non-redundant actuation), is [34]

$$
\ddot{\mathbf{y}}=\mathbf{F}(\mathbf{x})+\mathbf{G}_{\mathbf{0}}(\mathbf{x}) \mathbf{u}_{\mathbf{0}}
$$

where $\mathbf{x}=\left[q_{1}, \dot{q}_{1}, q_{2}, \dot{q}_{2}\right], \mathbf{y}=\left[q_{1}, q_{2}\right]^{T}, \mathbf{u}_{0}=\left[\tau_{1}, \tau_{2}\right]$, $\mathbf{G}_{\mathbf{0}}(\mathbf{x})=\mathbf{M}^{-1} \mathbf{F}(\mathbf{x})=-\mathbf{M}^{-1}\left(\begin{array}{cc}-h \dot{q}_{2} & -h\left(q_{1}+q_{2}\right) \\ h \dot{q}_{1} & 0\end{array}\right)$,

where $M$ is a $2 \times 2$ matrix whose expression can be found in [34].

Taking into account the torque expression in (61) and assuming that each link is actuated by two identical actuators, the dynamic model of the redundant manipulator can be written as

$$
\ddot{\mathbf{y}}=\mathbf{F}(\mathbf{x})+\mathbf{G}_{\mathbf{0}}(\mathbf{x}) \mathbf{R u}
$$

where $\mathbf{u}=\left[\tau_{11}, \tau_{12}, \tau_{21}, \tau_{22}\right]^{T}, \mathbf{R}=\left(\begin{array}{cccc}1 & 1 & 0 & 0 \\ 0 & 0 & 1 & 1\end{array}\right)$.

From (63), we see that the control gain matrix satisfies assumption 2, i.e. $\mathbf{G}(\mathbf{x})=\mathbf{G}_{\mathbf{0}}(\mathbf{x}) \mathbf{R}$. The actuation scheme is chosen as

$$
\mathbf{u}=\mathbf{R}^{T} \mathbf{v}_{\mathbf{0}}, \mathbf{v}_{\mathbf{0}}=\left[\tau_{01}, \tau_{02}\right]^{T}
$$

where $\tau_{01}, \tau_{02}$ are the nominal torques that are to be designed and distributed among the corresponding redundant joints.

For the simulation, the numerical values of the robot manipulator are taken as in [34], the initial state vector is $\mathbf{x}(0)=[0.5,0,0.25,0]^{T}$. The desired trajectories are $y_{d 1}=\sin (t)$ and $y_{d 2}=\cos (t)$. The design parameters are: $\lambda_{1}=\lambda_{2}=1, \quad \mathbf{K}=\operatorname{diag}\{1,1\}, \quad \mathbf{K}_{0}=\operatorname{diag}\{5,5\}$, $\varepsilon_{0}=0.01, \eta=0.001$. The initial value of $\boldsymbol{\theta}$ is zero, $\quad \mathbf{z}=\left[e_{1}, \dot{e}_{1}, e_{2}, \dot{e}_{2}\right]$.The fuzzy system uses three membership functions defined by:

$$
\begin{aligned}
& \mu_{F_{j}^{1}}\left(z_{j}\right)=\exp \left(-0.5\left(\left(z_{j}+0.25\right) / 0.6\right)^{2}\right) \\
& \mu_{F_{j}^{2}}\left(z_{j}\right)=\exp \left(-0.5\left(z_{j} / 0.6\right)^{2}\right) \\
& \mu_{F_{j}^{3}}\left(z_{j}\right)=\exp \left(-0.5\left(\left(z_{j}+0.25\right) / 0.6\right)^{2}\right) .
\end{aligned}
$$

We consider the two following failure scenarios:

\section{Failure scenario 1:}

For the first link, the first actuator is intact, while the second actuator hasundergone a type (4) failure at time $t=12 \mathrm{~s}$. For the second link, we suppose that the first actuator starts losing effectiveness exponentially at time $t=25 \mathrm{~s}$ (type (6) failure), while the second actuator gets locked in place at time $t=8 \mathrm{~s}$, then recovers from the failure at time $t=18 \mathrm{~s}$. This can be expressed as follows: $\bar{\tau}_{12}(t)=3.5, \bar{\tau}_{22}(t)=2.5$, $\rho_{21}(t)=e^{-(t-25)}$.

\section{Failure scenario 2:}

The first link, the first actuator remains intact, while the second actuator has undergone a type (4) failure with a time varying pattern, at time $t=15 \mathrm{~s}$. For the second link, we suppose that, at time $t=20 \mathrm{~s}$, the first actuator becomes only $80 \%$ effective (type (6) failure), while the second actuator gets locked in place at $t=10 \mathrm{~s}$. This is expressed as: $\bar{\tau}_{12}=2.5+0.1 \cos (2 t)+0.3 \sin (2 t)$, $\bar{\tau}_{22}(t)=2.5$, and $\rho_{21}(t)=0.8$.

The simulation is carried out for $30 \mathrm{~s}$ in both scenarios. The simulation results for the first scenario are shown in Figures $2-5$, and the results for the second scenario are shown in Figures 6-9. It can be seen that the proposed actuator failure compensation controller was able to meet the control objectives despite the presence of type (4) and type (6) failures. Besides, from the second scenario, if an actuator recovers from a failure, it will be automatically exploited by the controller to drive the link. In summary, the simulation results confirm the theoretical developments.

Remark 3. An interesting feature of this scheme is that it does not require an FDI module, which makes it more effective for real-time implementation, as the FDI module induces some latency with the system.
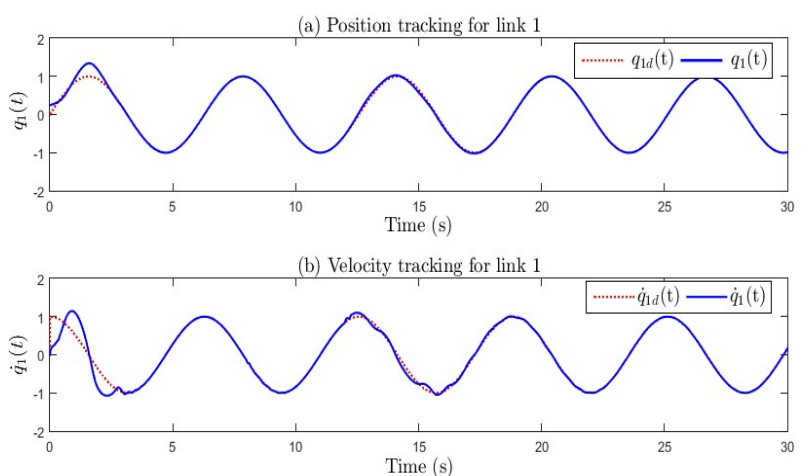

Figure 2. Tracking curves for link1 (scenario 1) 


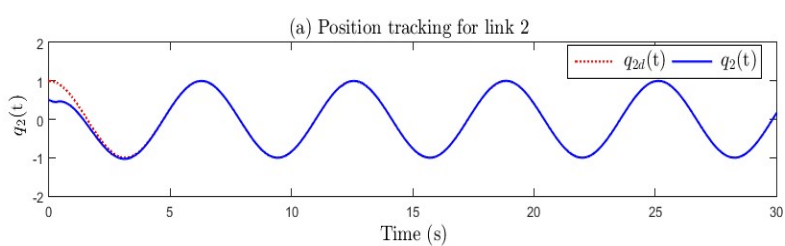

(b) Velocity tracking for link 2

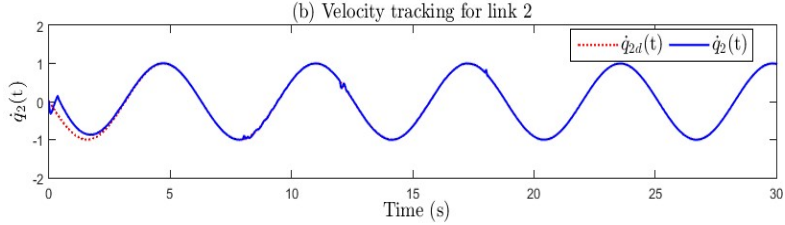

Figure 3. Tracking curves for link2 (scenario1)

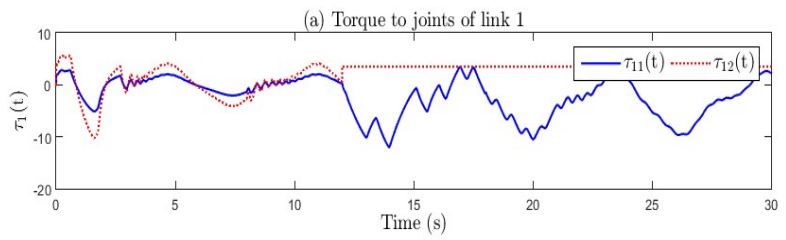

(b) Torque applied to joints of link 2

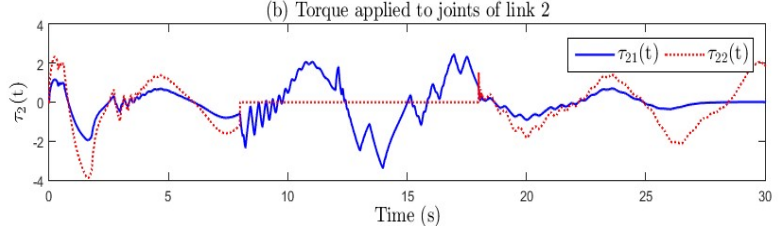

Figure 4. Applied torque curves (scenario 1)
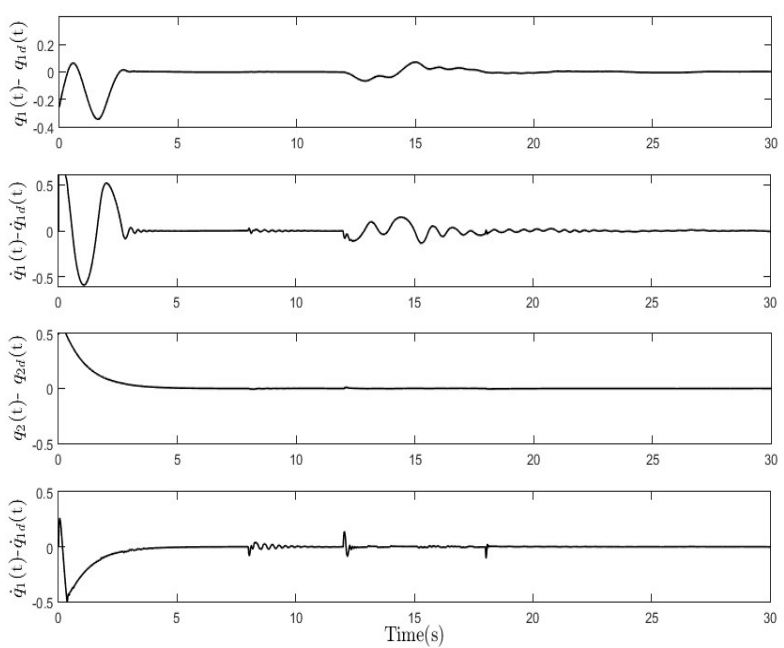

Figure 5. Tracking errors curves (scenario 1)
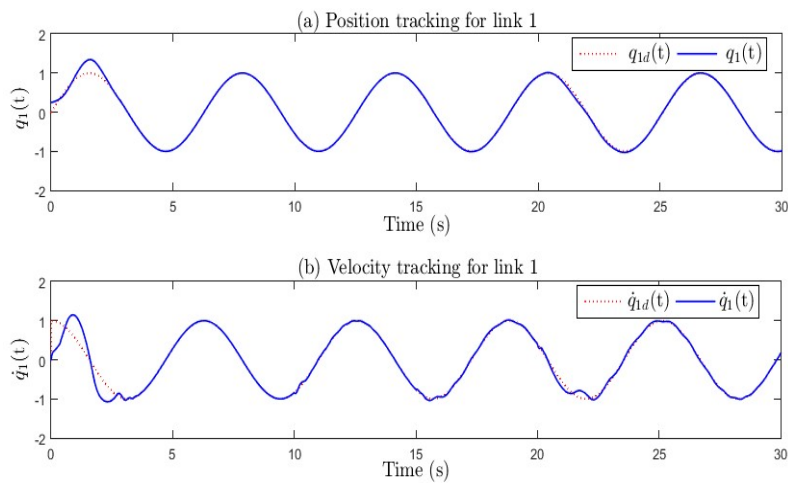

Figure 6. Tracking curves for link1 (scenario 2)
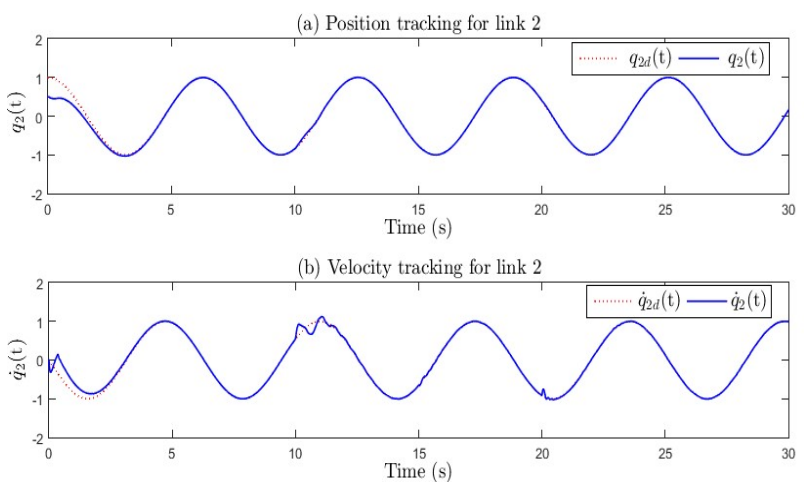

Figure 7. Tracking curves for link2 (scenario 2)
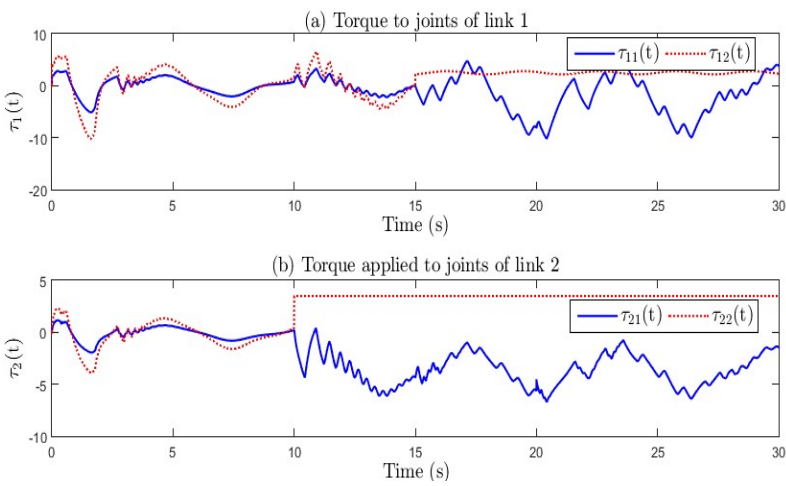

Figure 8. Applied torque curves (scenario 2)
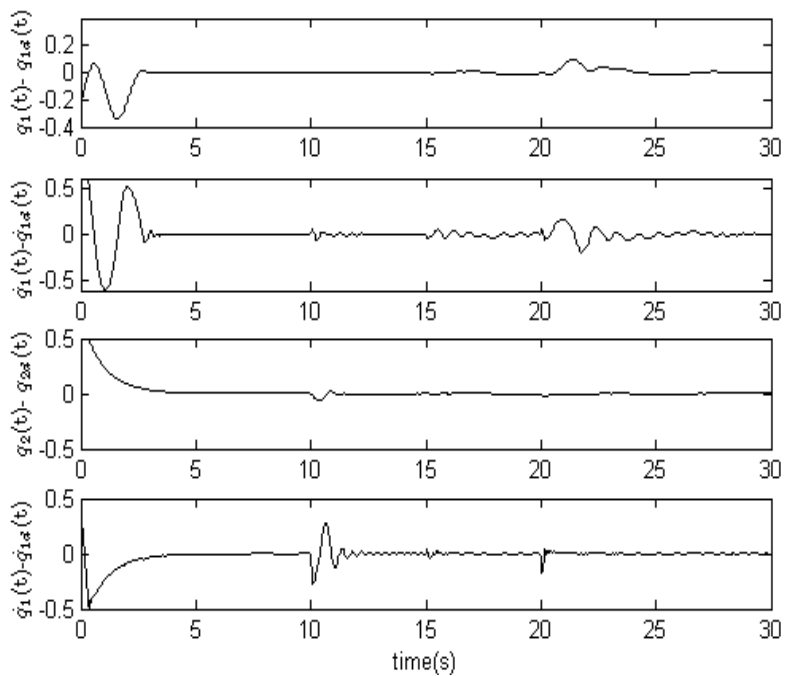

Figure 9. Tracking errors curves (scenario 2)

\section{CONCLUSION}

A stable adaptive actuator failure compensation control scheme was proposed for a class of nonlinear MIMO systems with redundant actuators. Under some mildassumptions on the system structure and available information, we were able to deal with two types of failures in a general framework, i.e. time varying actuator pattern and time-varying loss of effectiveness. In this scheme, we incorporated an adaptive fuzzy controller that deals with unknown system parameters, unknown actuator failures and robustness issue simultaneously. By using a 
piecewise Lyapunov analysis, we proofed that the controller meets the design specifications in the presence of actuator failures. The simulation on a dual joint two-link manipulator with possible joint failures shows the effectiveness and feasibility of the proposed scheme. Future works will focus on the introduction of state observers in the control loop to construct the states. Besides, we can also seek more relaxed design conditions and enlarge the set of actuator failures.

\section{References}

[1] Y. Zhang and J. Jiang, "Bibliographical review on reconfigurable fault-tolerant control systems," Annu. Rev. Control, vol. 32, no. 2, pp. 229-252, Dec. 2008, doi: 10.1016/j.arcontrol.2008.03.008.

[2] M. Blanke, M. Kinnaert, J. Lunze, and M. Staroswiecki, Diagnosis and Fault-Tolerant Control. Berlin, Heidelberg: Springer, 2016.

[3] L. Y. Wen, G. Tao, and H. Yang, "LQ control based actuator failure compensation," Optim. Control Appl. Methods, vol. 37, no. 2, pp. 227247, Mar. 2016, doi: 10.1002/oca.2162.

[4] A. Zhang, Y. Wang, Z. Zhang, and H. R. Karimi, "Robust control allocation for spacecraft attitude stabilization under actuator faults and uncertainty," Math. Probl. Eng., vol. 2014, 2014, doi: $10.1155 / 2014 / 789327$.

[5] F. Liao, J. J. L. Wang, and G. G. H. G. Yang, "Reliable robust flight tracking control: An LMI approach," IEEE Trans. Control Syst. Technol., vol. 10, no. 1, pp. 76-89, 2002, doi: 10.1109/87.974340.

[6] M. Yajie, J. Bin, and T. Gang, "A new multiplemodel adaptive actuator failure compensation scheme for a class of nonlinear MIMO systems," in 2015 34th Chinese Control Conference, CCC, Sep. 2015, vol. 2015-Septe, pp. 6274-6279, doi: 10.1109/ChiCC.2015.7260624.

[7] S. Ismail, A. A. Pashilkar, R. Ayyagari, and N. Sundararajan, "Improved neural-aided sliding mode controller for autolanding under actuator failures and severe winds," Aerosp. Sci. Technol., vol. 33, no. 1, pp. 55-64, 2014, doi: 10.1016/j.ast.2013.12.016

[8] P. Li and G.-H. Yang, "Control of uncertain nonlinear systems against actuator faults using adaptive fuzzy approximation," in 2009 American Control Conference, 2009, pp. 4446-4451, doi: 10.1109/ACC.2009.5160349.

[9] P. Li and G. Yang, "An adaptive fuzzy design for fault-tolerant control of MIMO nonlinear uncertain systems," J. Control Theory Appl., vol. 9, no. 2, pp. 244-250, May 2011, doi: 10.1007/s11768011-8167-x.

[10] S. Boulouma, S. Labiod, and H. Boubertakh, "Adaptive fuzzy actuator failure compensation for a class of nonlinear systems," in 2015 4th International Conference on Electrical Engineering, ICEE 2015, Dec. 2016, pp. 1-7, doi: 10.1109/INTEE.2015.7416722.

[11] S. Amezquita, W. Butt, and Y. Lin, "An adaptive dynamic surface control scheme for a class of output feedback nonlinear systems with actuator failures," in 2010 8th Wold Congress on Intelligent Control and Automation (WCICA), 2010, pp. 750755, doi: 10.1109/WCICA.2010.5554062.

[12] S. K. Amezquita, L. Yan, and W. A. Butt, "Adaptive dynamic surface control for a class of MIMO nonlinear systems with actuator failures," Int. J. Syst. Sci., vol. 44, no. 3, pp. 479-492, Mar. 2013, doi: 10.1080/00207721.2011.604740.

[13] T. Rugthum and G. Tao, "An adaptive actuator failure compensation scheme for a cooperative manipulator system," Robotica, vol. 34 , no. 07, pp. 1529-1552, 2016, doi: 10.1017/S0263574714002434.

[14] Y. J. Ma, B. Jiang, G. Tao, and Y. H. Cheng, "A direct adaptive actuator failure compensation scheme for satellite attitude control systems," Proc. Inst. Mech. Eng. Part G-Journal Aerosp. Eng., vol. 228, no. 4, pp. 542-556, 2014, doi: Doi 10.1177/0954410013476191.

[15] X. Yao, G. Tao, Y. Ma, and R. Qi, "Adaptive actuator failure compensation design for spacecraft attitude control," IEEE Trans. Aerosp. Electron. Syst., vol. 52, no. 3, pp. 1021-1034, Jun. 2016, doi: 10.1109/TAES.2016.130802.

[16] X. Yao, G. Tao, and B. Jiang, "Adaptive actuator failure compensation for multivariable feedback linearizable systems," Int. J. Robust Nonlinear Control, vol. 26, no. 2, pp. 252-285, Jan. 2016, doi: 10.1002/rnc.3309.

[17] Y. Yang and D. Yue, "Adaptive decentralized fault-tolerant tracking control of a class of uncertain large-scale nonlinear systems with actuator faults," Trans. Inst. Meas. Control, Nov. 2016, doi: 10.1177/0142331216670452.

[18] G. Tao, S. Chen, X. Tang, and S. M. Joshi, Adaptive Control of Systems with Actuator Failures. London: Springer London, 2004.

[19] G. Tao, "Direct adaptive actuator failure compensation control: a tutorial," J. Control Decis., vol. 1, no. 1, pp. 75-101, Jan. 2014, doi: 10.1080/23307706.2014.885292.

[20] S. Chen, G. Tao, and S. M. Joshi, "Adaptive Actuator Failure Compensation Designs for Linear Systems," Int. J. Control. Autom. Syst., vol. 2, no. 1, pp. 1-14, 2004.

[21] X. Tang, G. Tao, and S. M. Joshi, "Adaptive output feedback actuator failure compensation for a class of non-linear systems," Int. J. Adapt. Control Signal Process., vol. 19, no. 6, pp. 419444, 2005, doi: 10.1002/acs.843.

[22] W. Wang and C. Wen, "Adaptive actuator failure compensation control of uncertain nonlinear systems with guaranteed transient performance," Automatica, vol. 46, no. 12, pp. 2082-2091, Dec. 2010, doi: 10.1016/j.automatica.2010.09.006.

[23] P. Li and G. Yang, "Backstepping adaptive fuzzy control of uncertain nonlinear systems against actuator faults," J. Control Theory Appl., vol. 7, no. 3, pp. 248-256, Aug. 2009, doi: 10.1007/s11768-009-8074-6.

[24] X. Tang, G. Tao, and S. M. Joshi, "Adaptive actuator failure compensation for nonlinear MIMO systems with an aircraft control application," Automatica, vol. 43, no. 11, pp. 1869-1883, Nov. 
2007, doi: 10.1016/j.automatica.2007.03.019.

[25] Y. Ma, B. Jiang, G. Tao, and Y. Cheng, "Actuator failure compensation and attitude control for rigid satellite by adaptive control using quaternion feedback," J. Franklin Inst., vol. 351, no. 1 , pp. 296-314, 2014, doi: 10.1016/j.jranklin.2013.08.028.

[26] E. F. Kececi, X. Tang, and G. Tao, "Adaptive actuator failure compensation for redundant manipulators," Robotica, vol. 27, no. 01, pp. 1928, Jan. 2009, doi: $10.1017 /$ S0263574708004487.

[27] J. J. Slotine and W. Li, Applied Nonlinear
Control. Englewood Cliffs, NJ :Prentice Hall, 1991.

[28] S. Labiod and T. M. Guerra, "Direct adaptive fuzzy control for a class of MIMO nonlinear systems," Int. J. Syst. Sci., vol. 38, no. 8, pp. 665675, Aug. 2007, doi: 10.1080/00207720701500583.

[29] M. M. Polycarpou and P. A. loannou, "A robust adaptive nonlinear control design," Automatica, vol. 32, no. 3, pp. 423-427, Mar. 1996, doi: 10.1016/0005-1098(95)00147-6. 